\title{
Interplay between the factorization of the Jarlskog invariant and location of the solar and atmospheric resonances for neutrino oscillations in matter
}

\author{
Stephen J. Parke $\odot^{*}$ \\ Theoretical Physics Department, Fermi National Accelerator Laboratory, Batavia, Illinois 60510, USA
}

(Received 15 December 2020; accepted 19 January 2021; published 16 February 2021)

\begin{abstract}
The Jarlskog invariant which controls the size of intrinsic $C P$ violation in neutrino oscillation appearance experiments is modified by Wolfenstein matter effects for neutrinos propagating in matter. In this paper we give the exact factorization of the Jarlskog invariant in matter into the vacuum Jarlskog invariant times two, two-flavor matter resonance factors that control the matter effects for the solar and atmospheric resonances independently. We compare the location of the minima of the factorizing resonance factors with the location of the solar and atmospheric resonances, precisely defined. They are not identical but the fractional differences are both found to be less than $0.1 \%$. In addition, we explain why symmetry polynomials of the square of the mass of the neutrino eigenvalues in matter, such as inverse of the square of the Jarlskog invariant in matter, can be given as polynomials in the matter potential.
\end{abstract}

DOI: 10.1103/PhysRevD.103.033003

\section{INTRODUCTION}

In the current and future long baseline experiments, T2K [1], NOvA [2], DUNE [3] and T2HK(K) [4,5], neutrinos propagate between 300 to $1300 \mathrm{~km}$ through Earth's crust between source and detector. Neutrino oscillations in matter are significantly different than in vacuum because of the coherent forward scattering of the neutrinos off the electrons in matter. This effect is known as the Wolfenstein matter effect [6].

The neutrino mass eigenvalues and the mixing angles of the neutrinos in matter are strongly impacted by the matter effect which depends on density of the matter and the energy of the neutrino through the Wolfenstein matter potential [6], $a$, given by

$$
a \equiv 2 \sqrt{2} G_{F} N_{e} E_{\nu},
$$

where $G_{F}$ is the Fermi constant, $N_{e}$ is the number density of electrons, and $E_{\nu}$ is the neutrino energy in the matter rest frame. These changes in the masses and the mixing angles have a significant effect on the oscillation probabilities as the neutrinos transverse through matter.

One of the primary goals of the above long baseline accelerator experiments is to measure intrinsic $C P$ violation in the neutrinos sector. In vacuum, the $C P$-violating part of

\footnotetext{
parke@fnal.gov
}

Published by the American Physical Society under the terms of the Creative Commons Attribution 4.0 International license. Further distribution of this work must maintain attribution to the author(s) and the published article's title, journal citation, and DOI. Funded by SCOAP ${ }^{3}$. the neutrino oscillation probability in the appearance channels, e.g., $\nu_{\mu} \rightarrow \nu_{e}$, is given by [7]

$$
8 J \sin \Delta_{31} \sin \Delta_{32} \sin \Delta_{21},
$$

where the kinematic phases are given by $\Delta_{j k}=$ $\Delta m_{j k}^{2} L / 4 E_{\nu}$ with $\Delta m_{j k}^{2}=m_{j}^{2}-m_{k}^{2}$ for an experiment of baseline $L$ and neutrino energy $E_{\nu} . J$ is the Jarlskog invariant [8], that controls the size of $C P$ violation. Using the standard parametrization of the Pontecorvo, Maki, Nakagawa and Sakata (PMNS) matrix [9,10], the Jarlskog invariant is given by

$$
J \equiv s_{23} c_{23} s_{13} c_{13}^{2} s_{12} c_{12} \sin \delta
$$

where $s_{i j}=\sin \theta_{i j}, c_{i j}=\cos \theta_{i j}$.

For neutrinos propagating in matter, the part of the appearance oscillation probability that depends on the intrinsic $C P$ violation is given by

$$
8 \hat{J} \sin \hat{\Delta}_{31} \sin \hat{\Delta}_{32} \sin \hat{\Delta}_{21}
$$

where $\hat{x}$ is the matter value for the vacuum variable $x$. The Jarlskog invariant in matter, $\hat{J}$, is given by same expression as Eq. (3) but with the mixing angles and phase replaced by their matter values [11-13]. The variables $\hat{\theta}_{12}, \hat{\theta}_{13}$ and $\Delta \bar{m}^{2}{ }_{j k}$, that appear in Eq. (4), have a strong dependence on the Wolfenstein matter potential, $a$.

In [14], by Denton and Parke (DP), a simple and precise factorization of the Jarlskog invariant in matter was given as follows: 


$$
\hat{J} \approx \frac{J}{\mathcal{R}_{\odot}^{\mathrm{appx}} \mathcal{R}_{\mathrm{atm}}^{\mathrm{appx}}},
$$

where

$$
\begin{aligned}
& \mathcal{R}_{\odot}^{\mathrm{appx}}=\sqrt{1-2 c_{13}^{2} \cos 2 \theta_{12}\left(a / \Delta m_{21}^{2}\right)+c_{13}^{4}\left(a / \Delta m_{21}^{2}\right)^{2}}, \\
& \mathcal{R}_{\mathrm{atm}}^{\mathrm{appx}}=\sqrt{1-2 \cos 2 \theta_{13}\left(a / \Delta m_{e e}^{2}\right)+\left(a / \Delta m_{e e}^{2}\right)^{2}} .
\end{aligned}
$$

The fractional precision of this factorization is better than $0.07 \%$, for all $a$ and both mass orderings. A factorization with a precision of a few percent was given in [15] with a follow up paper, [16], that reproduced the precise factorization of DP. In this paper we give an extension of the analysis of DP to derive an exact factorization of this Jarlskog invariant in matter as well as a perturbative expansion in the two small quantities $s_{13}^{2}$ and $\Delta m_{21}^{2} / \Delta m_{e e}^{2}$.

Then, after defining precisely and calculating the location of the solar and atmospheric resonances to the relevant accuracy in the matter potential for the first time, we compare the location of the resonance with the location of the minima of the factorizing two flavor resonance factors. Although they are identical at the order given by DP, what is shown here is they are not identical at higher orders but the fractional differences are found to be small, less than $0.1 \%$. A qualitative understanding of why they differ is also given.

As part of our analysis, we show the simplicity of variables that can be expressed as a symmetric polynomial of the neutrino eigenvalues in matter, $\widehat{m^{2}}{ }_{j}$, such as the Jarlskog invariant in matter as well as other examples in Sec. II. We give the exact factorization of the Jarlskog invariant in matter as a product of two, two flavor resonance factors in Sec. III as well as a perturbative expansion. In Sec. IV, we compare the minima of these exact factorizing factors to the actual location of the solar and atmospheric resonance for the matter potential, followed by a summary and conclusion section. There are five appendixes that contain computational details. Thus this paper provides additional understanding of the Wolfenstein matter effect for three neutrinos propagating through matter.

\section{SIMPLICITY OF SYMMETRIC POLYNOMIALS OF THE EIGENVALUES}

In the flavor basis, the neutrino propagation Hamiltonian in matter is given by

$$
(2 E) H=U\left(\begin{array}{ccc}
0 & & \\
& \Delta m_{21}^{2} & \\
& & \Delta m_{31}^{2}
\end{array}\right) U^{\dagger}+\left(\begin{array}{ccc}
a & & \\
& 0 & \\
& & 0
\end{array}\right) .
$$

$U$ is the PMNS $[9,10]$ lepton mixing matrix, parametrized by

$$
\begin{aligned}
U \equiv & U_{23}\left(\theta_{23}, \delta\right) U_{13}\left(\theta_{13}\right) U_{12}\left(\theta_{12}\right) \\
\equiv & \left(\begin{array}{ccc}
1 & & \\
& c_{23} & s_{23} e^{i \delta} \\
-s_{23} e^{-i \delta} & c_{23}
\end{array}\right)\left(\begin{array}{ccc}
c_{13} & & s_{13} \\
& 1 & \\
-s_{13} & & c_{13}
\end{array}\right) \\
& \times\left(\begin{array}{ccc}
c_{12} & s_{12} & \\
-s_{12} & c_{12} & \\
& & 1
\end{array}\right)
\end{aligned}
$$

where $s_{i j}=\sin \theta_{i j}$ and $c_{i j}=\cos \theta_{i j}$ are given by

$$
\begin{aligned}
& \sin ^{2} \theta_{13} \equiv\left|U_{e 3}\right|^{2} \approx 0.022, \\
& \sin ^{2} \theta_{12} \equiv\left|U_{e 2}\right|^{2} /\left(1-\left|U_{e 3}\right|^{2}\right) \approx 0.32, \\
& \sin ^{2} \theta_{23} \equiv\left|U_{\mu 3}\right|^{2} /\left(1-\left|U_{e 3}\right|^{2}\right) \approx 0.55 .
\end{aligned}
$$

The sine and cosine of $\delta$ are given by

$e^{i \delta} \equiv \frac{\left(1-\left|U_{e 3}\right|^{2}\right) U_{e 2} U_{\mu 2}^{*} U_{e 3}^{*} U_{\mu 3}+\left|U_{e 2}\right|^{2}\left|U_{e 3}\right|^{2}\left|U_{\mu 3}\right|^{2}}{\left|U_{e 1} U_{e 2} U_{e 3} U_{\mu 3} U_{\tau 3}\right|}$.

At this time, circa 2020, the numerical value of $\delta$ is still to be determined. Our definition of the $C P$-violating phase $\delta$ is invariant under rephasing ${ }^{1}$ of the rows and columns of $U$ and thus shifting it from its PDG location, i.e., from next to $s_{13}$ to next to $s_{23}$, does not affect any observable. ${ }^{2}$ This choice is made because the $\theta_{23}, \delta$ submatrix of $U$ commutes with the matter potential and factors these variables from many expressions. For other choices for the ordering of the factorization of the PMNS matrix see [17].

For the long baseline neutrino oscillation experiments, $\mathrm{T} 2 \mathrm{~K}$, NOvA, DUNE, and $\mathrm{T} 2 \mathrm{HK}(\mathrm{K})$ it is sufficient to consider the matter density along the path of the neutrino to be a constant, $a$, as has been discussed in detail in $[18,19]$. Therefore, for the rest of this paper we will consider the matter potential to be a constant for a neutrino of a given energy. This simplifies the solution to the evolution of the neutrino state in matter significantly, by allowing for exact analytical solution. However, the resulting solution is still analytically impenetrable as we will see later in this section.

The characteristic equation for the matrix $(2 \mathrm{E}) \mathrm{H}$ gives the eigenvalues of the square of the neutrino masses in matter, $\widehat{m^{2}}{ }_{j}$, all satisfying

$$
\left({\widehat{m^{2}}}_{j}\right)^{3}-A\left({\widehat{m^{2}}}_{j}\right)^{2}+B{\widehat{m^{2}}}_{j}-C=0,
$$

\footnotetext{
${ }^{1}$ The commonly used definition, $\delta=-\operatorname{Arg}\left(U_{e 3}\right)$, is not a rephasing invariant and requires an explicit phase choice especially in the $U_{\alpha j}$ elements, for $\alpha=\mu$ and $\tau, j=1$ and 2 .

${ }^{2}$ The sign of $\delta$ given by Eq. (10) is consistent with the sign in the PDG expression for PMNS matrix.
} 
where $A, B$, and $C$ are the sum of the eigenvalues, sum of the products of the eigenvalues, and the triple product of the eigenvalues:

$$
\begin{aligned}
A & \equiv \sum_{j} \widehat{m^{2}}{ }_{j}=\Delta m_{31}^{2}+\Delta m_{21}^{2}+a, \\
B & \equiv \sum_{j>k} \widehat{m^{2}{ }_{j} \widehat{m}^{2}}{ }_{k} \\
& =\Delta m_{31}^{2} \Delta m_{21}^{2}+a\left(\Delta m_{31}^{2} c_{13}^{2}+\Delta m_{21}^{2}\left(c_{12}^{2}+s_{13}^{2} s_{12}^{2}\right)\right), \\
C & \equiv \prod_{j} \widehat{m^{2}}{ }_{j}=a \Delta m_{31}^{2} \Delta m_{21}^{2} c_{13}^{2} c_{12}^{2} .
\end{aligned}
$$

We use the convention that in vacuum $\left(\widehat{m^{2}}{ }_{1}, \widehat{m}^{2}{ }_{2}, \widehat{m^{2}}{ }_{3}\right)=$ $\left(0, \Delta m_{21}^{2}, \Delta m_{31}^{2}\right)$.

From Refs. [11,20,21], the exact eigenvalues in matter are

$$
\begin{aligned}
& \widehat{m^{2}}{ }_{1}=\frac{1}{3} A-\frac{1}{3} \sqrt{A^{2}-3 B}\left(\mathcal{Z}+\sqrt{3} \sqrt{1-\mathcal{Z}^{2}}\right), \\
& \widehat{m^{2}}{ }_{2}=\frac{1}{3} A-\frac{1}{3} \sqrt{A^{2}-3 B}\left(\mathcal{Z}-\sqrt{3} \sqrt{1-\mathcal{Z}^{2}}\right), \\
& \widehat{m^{2}}{ }_{3}=\frac{1}{3} A+\frac{2}{3} \sqrt{A^{2}-3 B} \mathcal{Z},
\end{aligned}
$$

where $\mathcal{Z}$ contains the $\cos \left\{\frac{1}{3} \cos ^{-1}[\cdots]\right\}$ terms, given by

$$
\mathcal{Z}=\cos \left\{\frac{1}{3} \cos ^{-1}\left[\frac{2 A^{3}-9 A B+27 C}{2\left(A^{2}-3 B\right)^{3 / 2}}\right]+\zeta\right\},
$$

with $\zeta=0$ for normal ordering (NO) that gives $\widehat{m^{2}}{ }_{1}<$ $\widehat{m^{2}}{ }_{2}<{\widehat{m^{2}}}_{3}$ and $\zeta=2 \pi / 3$ for inverted ordering (IO) that gives $\widehat{m^{2}}{ }_{3}<\widehat{m}^{2}{ }_{1}<\widehat{m}_{2}^{2}$, the usual conventions. This convoluted term, $\cos \left\{\frac{1}{3} \cos ^{-1}[\cdots]\right\}$, which is a generic feature of the analytic solution to cubic equations, does not lend itself to a useful perturbative expansion for arbitrary values of the matter potential, even though, for neutrino oscillations there are two small parameters $\sin ^{2} \theta_{13} \sim 0.02$ and $\Delta m_{21}^{2} / \Delta m_{31}^{2} \sim 0.03$. This unfortunate fact ${ }^{3}$ makes the exact analytical solution, Eq. (13), only useful, in general, for numerical studies, not analytic understanding.

However, while the exact eigenvalues have a very complicated analytic form, any symmetric polynomial in the eigenvalues can be uniquely expressed in terms of $A, B$, and $C$. This follows from the fundamental theorem of symmetric polynomials (FTSP) [22] and allows any symmetric polynomial of the $\widehat{m^{2}}{ }_{i}$ to be written as a polynomial in the matter potential, substantially simplifying such expressions.

\footnotetext{
${ }^{3}$ As an example of the analytic impenetrability of $\mathcal{Z}$, setting $a=0$ and recovering the vacuum values for the eigenvalues, $\left(0, \Delta m_{21}^{2}, \Delta m_{31}^{2}\right)$, is a highly nontrivial exercise. In vacuum $\mathcal{Z}=$ $\frac{1}{2}\left(\Delta m_{31}^{2}+\Delta m_{32}^{2}\right) / \sqrt{\left(\Delta m_{32}^{2}\right)^{2}+\Delta m_{21}^{2} \Delta m_{31}^{2}}$ for NO.
}

An example of this is given in $[14,23,24]$,

$$
\begin{aligned}
\left(\prod_{j>k}{\widehat{\Delta m^{2}}}_{j k}\right)^{2}= & \left(A^{2}-4 B\right)\left(B^{2}-4 A C\right) \\
& +(2 A B-27 C) C
\end{aligned}
$$

which given the expressions for $A, B$, and $C$ in Eq. (12) is a fourth order polynomial in the matter potential, $a$. No impenetrable $\cos \left\{\frac{1}{3} \cos ^{-1}[\cdots]\right\}$ terms appear.

The eigenvector-eigenvalue identity $[25,26]$ gives us the elements of the PMNS matrix in matter as follows:

$$
\left|\hat{U}_{\alpha i}\right|^{2}=\frac{\left({\widehat{m^{2}}}_{i}\right)^{2}-(\xi+\chi)_{\alpha}{\widehat{m^{2}}}_{i}+(\xi \chi)_{\alpha}}{\Delta{\widehat{m^{2}}}{ }_{i j} \Delta \widehat{m}^{2}{ }_{i k}},
$$

where $i, j$, and $k$ are all different. The variables $(\xi+\chi)_{\alpha}$ and $(\xi \chi)_{\alpha}$, which are the trace and determinant, respectively, of (2E)H with the $\alpha$ row and $\alpha$ column removed. All $(\xi+\chi)_{\alpha}$ and $(\xi \chi)_{\alpha}$ are linear polynomials in the matter potential, $a$, and are given in Appendix A.

Combining Eqs. (15) and (16), we have that

$$
\begin{aligned}
& \left(\prod_{j>k} \Delta{\widehat{m^{2}}}_{j k}\right)^{2}\left(\prod_{i}\left|\hat{U}_{\alpha i}\right|^{2}\right) \\
& =\prod_{i}\left(\left(\widehat{m^{2}}{ }_{i}\right)^{2}-(\xi+\chi)_{\alpha} \widehat{m^{2}}{ }_{i}-(\xi \chi)_{\alpha}\right)
\end{aligned}
$$

is a symmetric polynomial in the eigenvalues, $\widehat{m^{2}}$, and thus can be written as a polynomial in $A, B, C,(\xi+\chi)_{\alpha}$ and $(\xi \chi)_{\alpha}$, as follows:

$$
\begin{aligned}
& \prod_{i}\left(\left(\widehat{m^{2}}{ }_{i}\right)^{2}-(\xi+\chi)_{\alpha} \widehat{m^{2}}{ }_{i}+(\xi \chi)_{\alpha}\right) \\
& =C\left[C-B(\xi+\chi)_{\alpha}+A(\xi+\chi)_{\alpha}^{2}-(\xi+\chi)_{\alpha}^{3}\right] \\
& \quad-(\xi+\chi)_{\alpha}(\xi \chi)_{\alpha}\left[(A B-3 C)-B(\xi+\chi)_{\alpha}+A(\xi \chi)_{\alpha}\right] \\
& \quad+(\xi \chi)_{\alpha}\left[\left(B^{2}-2 A C\right)+\left(A^{2}-2 B\right)(\xi \chi)_{\alpha}+(\xi \chi)_{\alpha}^{2}\right] .
\end{aligned}
$$

This is also a polynomial in the matter potential of maximum fourth order. In Appendix B we give this expression which is straightforward to evaluate for $\alpha=e, \mu$, and $\tau$. Again, no $\cos \left\{\frac{1}{3} \cos ^{-1}[\cdots]\right\}$ terms appear.

For $\alpha=e$ for Eq. (17), one finds that the right-hand side is independent of the matter potential, i.e.,

$$
\begin{aligned}
& \left(\prod_{j>k} \Delta{\widehat{m^{2}}}_{j k}\right)^{2}\left(\prod_{i}\left|\hat{U}_{e i}\right|^{2}\right) \\
& =\left(\prod_{j>k} \Delta m^{2}{ }_{j k}\right)^{2}\left(\prod_{i}\left|U_{e i}\right|^{2}\right) .
\end{aligned}
$$


This is the well-known Naumov-Harrison-Scott identity [23,27], divided by the Toshev identity [28], squared. In this form, this invariance is not a surprise as in the $a \rightarrow+\infty$ limit

$$
\begin{aligned}
\left(\prod_{j>k} \widehat{\Delta m^{2}}{ }_{j k}\right)^{2} & \rightarrow a^{4}, \\
\left|\hat{U}_{e i}\right|^{2} & \rightarrow \frac{1}{a^{2}}\left\{\begin{array}{ll}
i=1,2 & \text { for NO } \\
i=1,3 & \text { for IO }
\end{array} .\right.
\end{aligned}
$$

This implies that the fourth order polynomial must just be a constant.

For $\alpha=\mu$ or $\tau$, again in the $a \rightarrow+\infty$ limit

$$
\left|\hat{U}_{\mu i}\right|^{2} \rightarrow \frac{1}{a^{2}}\left\{\begin{array}{ll}
i=3 & \text { for } \mathrm{NO} \\
i=2 & \text { for IO }
\end{array} .\right.
$$

This implies that the right-hand side of Eq. (17) is a quadratic polynomial in the matter potential, $a$. This fact is confirmed by explicit calculation where the coefficients of the powers the matter potential $a$, depend on $\theta_{23}$ and $\cos \delta$. Further details are also given in Appendix B.

\section{EXACT JARLSKOG INVARIANT IN MATTER}

We start from the exact Naumov-Harrison-Scott identity $[23,27]$, which is that the Jarlskog factor in matter times the product of the $\Delta m^{2}$ matter is an exact invariant ${ }^{4}$ :

$$
\frac{J}{\hat{J}}=\frac{\Delta \bar{m}_{32}{\widehat{m m^{2}}}_{31} \Delta{\widehat{m^{2}}}_{21}}{\Delta m_{32}^{2} \Delta m_{31}^{2} \Delta m_{21}^{2}} .
$$

While the exact eigenvalues have a very complicated analytic form [11] due to the presence of the $\cos \left\{\frac{1}{3} \cos ^{-1}[\cdots]\right\}$ terms, $J^{2} / \hat{J}^{2}$ can be written as a simple fourth order polynomial of the vacuum parameters and the matter potential, because of the FTSP.

Combining Eq. (12) and (15) one obtains the exact expression for $\left(\prod_{i>j} \Delta{\widehat{m^{2}}}_{i j}\right)^{2}$ as a fourth order polynomial in the matter potential, $a$. This guarantees its factorization in two quadratics as shown by Lodovico de Ferrari in 1540. We start by writing Eq. (20) as follows:

$$
\frac{J^{2}}{\hat{J}^{2}}=1+\sum_{n=1}^{4} f_{n}\left(\frac{a}{\Delta m_{21}^{2}}\right)^{n}
$$

where the $f_{n}$ 's are dimensionless functions of the vacuum oscillation parameters which can be easily derived from Eq. (15). Explicit expressions for the $f_{n}$ 's are given in

\footnotetext{
${ }^{4}$ The simplest way to "derive" this expression is that in the $L / E \rightarrow 0$, Eq. (2) and (4) must be equal.
}

Appendix C. $f_{1}$ and $f_{2}$ are of order 1 , where as $f_{3}$ is of order $\epsilon$ and $f_{4}$ is of order $\epsilon^{2}$, where

$$
\epsilon \equiv \Delta m_{21}^{2} / \Delta m_{e e}^{2} \sim 0.03,
$$

and $\Delta m_{e e}^{2} \equiv c_{12}^{2} \Delta m_{31}^{2}+s_{12}^{2} \Delta m_{31}^{2}$, [29]. Therefore, there is a distinct hierarchy in the $f_{n}$ 's.

Equation (21) can be exactly factorized as

$$
\begin{aligned}
\left(\frac{J}{\hat{J}}\right)^{2}= & \left(1-2 S_{\mathrm{sol}}^{\mathrm{ex}}\left(\frac{a}{\Delta m_{21}^{2}}\right)+T_{\mathrm{sol}}^{\mathrm{ex}}\left(\frac{a}{\Delta m_{21}^{2}}\right)^{2}\right) \\
& \times\left(1-2 S_{\mathrm{atm}}^{\mathrm{ex}}\left(\frac{a}{\Delta m_{e e}^{2}}\right)+T_{\mathrm{atm}}^{\mathrm{ex}}\left(\frac{a}{\Delta m_{e e}^{2}}\right)^{2}\right)
\end{aligned}
$$

with

$$
\begin{gathered}
S_{\mathrm{sol}}^{\mathrm{ex}}=\frac{1}{4}\left(-f_{1}+\sqrt{f_{1}^{2}+4\left(y-f_{2}\right)}\right), \\
T_{\mathrm{sol}}^{\mathrm{ex}}=\frac{1}{2}\left(y+\sqrt{y^{2}-4 f_{4}}\right), \\
\left.S_{\mathrm{atm}}^{\mathrm{ex}}=\frac{1}{4}\left(-f_{1}-\sqrt{f_{1}^{2}+4\left(y-f_{2}\right.}\right)\right) / \epsilon, \\
T_{\mathrm{atm}}^{\mathrm{ex}}=\frac{1}{2}\left(y-\sqrt{y^{2}-4 f_{4}}\right) / \epsilon^{2} .
\end{gathered}
$$

The $1 / \epsilon$ and $1 / \epsilon^{2}$ terms for $S_{\mathrm{atm}}^{\mathrm{ex}}$ and $T_{\mathrm{atm}}^{\mathrm{ex}}$, respectively, are needed to change the $\left(a / \Delta m_{21}^{2}\right)$ to $\left(a / \Delta m_{e e}^{2}\right)$ in the second brackets of Eq. (23). Except for the $a^{3}$ term, the coefficients for all other powers of $a$ are satisfied for any value of $y$. To get the correct coefficient for the $a^{3}$ terms, $y$ must satisfy the following cubic equation:

$$
\begin{aligned}
& y^{3}+g_{2} y^{2}+g_{1} y+g_{0}=0 \\
& \text { with } g_{2}=-f_{2}, \quad g_{1}=f_{1} f_{3}-4 f_{4} \\
& \text { and } g_{0}=\left(4 f_{2}-f_{1}^{2}\right) f_{4}-f_{3}^{2} .
\end{aligned}
$$

The relevant, exact solution to this cubic equation is

$$
\begin{aligned}
& y=2 \sqrt{Q} \cos \left\{\frac{1}{3} \arccos \left[R / \sqrt{Q^{3}}\right]\right\}-g_{2} / 3, \\
& Q=\left(g_{2}^{2}-3 g_{1}\right) / 9, \quad R=\left(9 g_{1} g_{2}-27 g_{0}-2 g_{2}^{3}\right) / 54,
\end{aligned}
$$

where $y \approx f_{2} \sim 1$. The other solutions give complex coefficients in the factorization of Eq. (23).

Unfortunately the solution to the cubic equation does not provide any analytic insight even though it is the exact solution. However, because the $f_{n}$ 's have a distinct hierarchy in $\Delta m_{21}^{2} / \Delta m_{e e}^{2}$, so do the $g_{n}$ 's: $g_{2}$ is of order 1 , whereas $g_{1}$ and $g_{0}$ are first and second order in 
TABLE I. The fractional differences for the coefficients of the factorizing quadratics between DP, [14], and the exact, Eqs. (24)-(27), (middle row) as well as between the perturbative expansions given in this paper Eqs. (32)-(35) and the exact (bottom row). For this table the following parameters where used: $\sin ^{2} \theta_{13}=0.022$, $\sin ^{2} \theta_{12}=0.32$, and $\Delta m_{21}^{2}=7.5 \times 10^{-5} \mathrm{eV}^{2} . \Delta m_{e e}^{2}= \pm 2.5 \times 10^{-3} \mathrm{eV}^{2}$ where the plus (minus) sign is for NO (IO). The matter density times electron fraction used is $Y_{e} \rho=1.5 \mathrm{~g} / \mathrm{cm}^{3}$.

\begin{tabular}{lcccc}
\hline \hline NO (IO) & $S_{\mathrm{sol}} / S_{\mathrm{sol}}^{\mathrm{ex}}-1$ & $T_{\mathrm{sol}} / T_{\mathrm{sol}}^{\mathrm{ex}}-1$ & $S_{\mathrm{atm}} / S_{\mathrm{atm}}^{\mathrm{ex}}-1$ & $T_{\mathrm{atm}} / T_{\mathrm{atm}}^{\mathrm{ex}}-1$ \\
\hline DP approx & $+1.9(-1.9) \times 10^{-3}$ & $+4.7(-5.0) \times 10^{-4}$ & $-1.9(-1.9) \times 10^{-4}$ & $-4.0(-4.0) \times 10^{-4}$ \\
Eq. (32) to (35) & $-1.6(+1.4) \times 10^{-7}$ & $-6.1(+2.5) \times 10^{-7}$ & $+2.8(+2.8) \times 10^{-7}$ & $-6.9(-6.9) \times 10^{-8}$ \\
\hline \hline
\end{tabular}

$\Delta m_{21}^{2} / \Delta m_{e e}^{2}$, respectively. This allows an iterative solution to Eq. (28),

$$
y^{(n)}=-g_{2}-g_{1} / y^{(n-1)}-g_{0} /\left(y^{(n-2)}\right)^{2}
$$

with $y^{(0)}=-g_{2}$ and $y^{(1)}=-\left(g_{2}^{2}-g_{1}\right) / g_{2}$. Therefore, $y$ can easily be calculated to the required order in $\Delta m_{21}^{2} / \Delta m_{e e}^{2}$.

Then to obtain the factorization coefficients, $S_{\text {sol }}$ thru $T_{\text {atm }}$, Eqs. (24)-(27), we use the fact that for neutrino oscillations there are two small parameters,

$$
\sin ^{2} \theta_{13} \sim 0.02 \text { and } \epsilon \equiv \Delta m_{21}^{2} / \Delta m_{e e}^{2} \sim 0.03
$$

so that one can easily perform a Taylor series expansion in these two small qualities to obtain

$$
\begin{aligned}
& S_{\mathrm{sol}} \approx c_{13}^{2} \cos 2 \theta_{12}-\left(s_{13}^{2} \epsilon\right)-\cos 2 \theta_{12}\left(s_{13}^{2} \epsilon^{2}\right)+\cdots, \\
& T_{\mathrm{sol}} \approx c_{13}^{4}-2 \cos 2 \theta_{12}\left(s_{13}^{2} \epsilon\right)+2 \cos 2 \theta_{12}\left(s_{13}^{4} \epsilon\right) \\
&- 2\left(1-6 s_{12}^{2} c_{12}^{2}\right)\left(s_{13}^{2} \epsilon^{2}\right)+\cdots, \\
& S_{\mathrm{atm}} \approx \cos 2 \theta_{13}+s_{12}^{2} c_{12}^{2}\left(\epsilon^{2}\right)-4 s_{12}^{2} c_{12}^{2}\left(s_{13}^{2} \epsilon^{2}\right) \\
& \quad+s_{12}^{2} c_{12}^{2} \cos 2 \theta_{12}\left(\epsilon^{3}\right)+\cdots, \\
& T_{\mathrm{atm}} \approx 1+2 s_{12}^{2} c_{12}^{2}\left(\epsilon^{2}\right)+2 s_{12}^{2} c_{12}^{2} \cos 2 \theta_{12}\left(\epsilon^{3}\right)+\cdots .
\end{aligned}
$$

The first term in each of these equations gives the approximation discussed in DP and the correction to the first term are of $\mathcal{O}\left(\epsilon^{2}\right)$ or $\mathcal{O}\left(s_{13}^{2} \epsilon\right)$ as expected.

In Table I we numerical evaluate the exact factorization along with those of DP and the approximation given by Eq. (32) to (35).

Thus the factorization of the Jarlskog invariant in matter is given by

$$
J \approx \mathcal{R}_{\odot} \mathcal{R}_{\mathrm{atm}} \hat{J}
$$

where

$$
\mathcal{R}_{\odot}=\sqrt{1-2 S_{\mathrm{sol}}\left(\frac{a}{\Delta m_{21}^{2}}\right)+T_{\mathrm{sol}}\left(\frac{a}{\Delta m_{21}^{2}}\right)^{2}},
$$

$$
\mathcal{R}_{\mathrm{atm}}=\sqrt{1-2 S_{\mathrm{atm}}\left(\frac{a}{\Delta m_{e e}^{2}}\right)+T_{\mathrm{atm}}\left(\frac{a}{\Delta m_{e e}^{2}}\right)^{2}} .
$$

This is the same functional form as in DP, Eq. (5). Depending on which approximation one uses for the $S$ 's and $T$ 's one obtains a fractional precision of $7 \times 10^{-4}$ using DP, Eq. (6), $4 \times 10^{-6}$ using Eqs. (32)-(35) and machine precision using the numerical solution to the cubic equation and Eqs. (24)-(27). See Fig. 1 for both the ratio of $\hat{J} / J$, as well as the fractional difference between the exact and the approximation of DP, Eq. (6) and that of Eqs. (32)-(35).

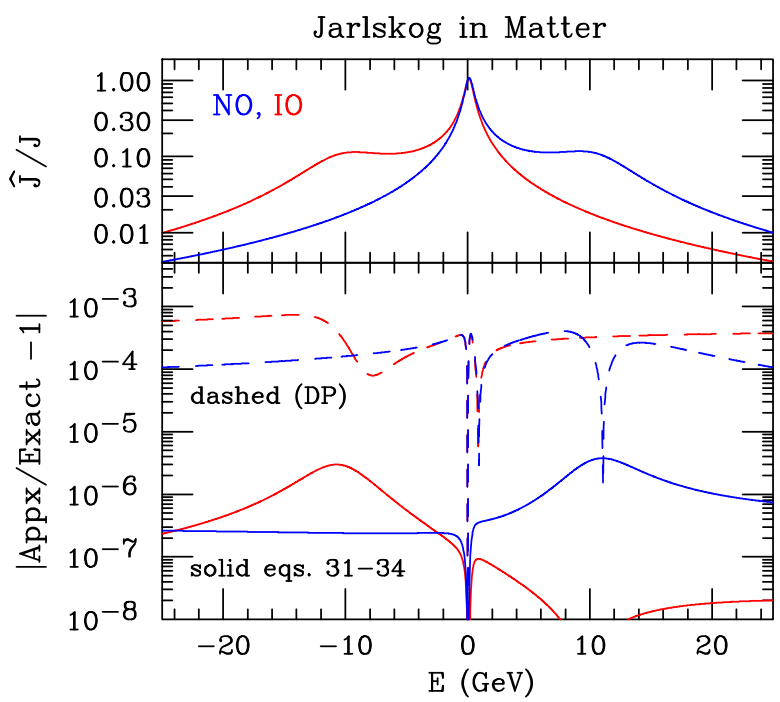

FIG. 1. Top: ratio between the Jarlskog invariant in matter and the vacuum Jarlskog invariant for both NO (blue) and IO (red) as a function of the energy of the neutrino. Here $Y_{e} \rho=1.5 \mathrm{~g} . \mathrm{cm}^{-3}$. Bottom: fractional difference between the various approximation to the factorization of the Jarlskog invariant compared with the exact Jarlskog invariant in matter. The dashed lines are using the DP approximation, Eq. (5) [14], whereas the solid lines are the third order approximation given in this paper, Eqs. (32)-(35), giving more than two orders of magnitude improvement. The results for the exact factorization, Eqs. (24)-(27), are not shown on this figure because they depend on the machine precision; using "double precision" one obtains $10^{-14}$ fractional precision. Positive (negative) energy is for neutrinos (antineutrinos) and zero is vacuum for both neutrinos and antineutrinos and all energies. 
If by machine precision one means "double precision," then the fractional difference is $\sim 10^{-14}$. For phenomenological purposes the precision using the simple result of DP [14] is accurate enough for most applications.

\section{RELATIONSHIP WITH THE SOLAR AND ATMOSPHERIC RESONANCES}

Its easily shown that $\mathcal{R}_{\odot}$ is minimum when ${ }^{5}$

$$
\begin{aligned}
\frac{a_{\mathrm{min}}^{\mathrm{sol}}}{\Delta m_{21}^{2}}=\frac{S_{\mathrm{sol}}^{\mathrm{ex}}}{T_{\mathrm{sol}}^{\mathrm{ex}}} & =\frac{\cos 2 \theta_{12}}{c_{13}^{2}}+\cos 4 \theta_{12}\left(s_{13}^{2} \epsilon\right)+\cdots \\
& =\frac{\cos 2 \theta_{12}}{c_{13}^{2}}+\left\{\begin{array}{ll}
-5.2 \times 10^{-4} & \mathrm{NO} \\
5.0 \times 10^{-4} & \mathrm{IO}
\end{array},\right.
\end{aligned}
$$

and that $\mathcal{R}_{\text {atm }}$ is minimum when

$$
\begin{aligned}
\frac{a_{\mathrm{min}}^{\mathrm{atm}}}{\Delta m_{e e}^{2}}=\frac{S_{\mathrm{atm}}^{\mathrm{ex}}}{T_{\mathrm{atm}}^{\mathrm{ex}}} & =\cos 2 \theta_{13}-s_{12}^{2} c_{12}^{2}\left(\epsilon^{2}\right)+\cdots \\
& =\cos 2 \theta_{13}- \begin{cases}2.0 \times 10^{-4} & \text { NO } \\
1.9 \times 10^{-4} & \text { IO }\end{cases}
\end{aligned}
$$

from Eqs. (37) and (38) respectively. The “..." are higher order terms in $s_{13}^{2}$ and $\epsilon$. Clearly, these two values for the matter potential are close to the values for the solar and atmospheric resonances. But what is the precise relationship? It cannot be exact as both $\mathcal{R}_{\odot}$ and $\mathcal{R}_{\text {atm }}$ are symmetric functions about the value that minimizes them, whereas for the solar and atmospheric resonances there must be some asymmetry caused by the fact that the solar resonance is below (above) atmospheric resonance for the NO (IO).

To answer this question we first have to define the solar and atmospheric resonance. For the solar resonance we define it to be the value of the matter potential that minimizes the separation between the matter mass eigenstates $\left|\hat{\nu}_{1}\right\rangle$ and $\left|\hat{\nu}_{2}\right\rangle$, that is when

$$
\frac{d\left(\widehat{m^{2}}{ }_{2}-\widehat{m^{2}}\right)}{d a}=0
$$

It can be easily shown by taking the derivative of $A, B$, and $C$ with respect to $a$, see Appendix D, or using the evolution equations of [30] that

$$
\frac{\widehat{d m^{2}}}{d a}=\left|\hat{U}_{e i}\right|^{2}
$$

Therefore the solar resonance condition also implies that

$$
\left|\hat{U}_{e 1}\right|^{2}=\left|\hat{U}_{e 2}\right|^{2}, \quad \sin ^{2} \hat{\theta}_{12}=0.5
$$

\footnotetext{
${ }^{5}$ For the numerical calculations in this section, we use the same parameters as in Table I.
}

That is, by minimizing the separation of the matter mass eigenstates $\left|\hat{\nu}_{1}\right\rangle$ and $\left|\hat{\nu}_{2}\right\rangle$, we also have exact maximal mixing between the matter eigenstates $\left|\hat{\nu}_{1}\right\rangle$ and $\left|\hat{\nu}_{2}\right\rangle$.

For the atmospheric resonance, one could consider the resonance condition for $\mathrm{NO}$ to be

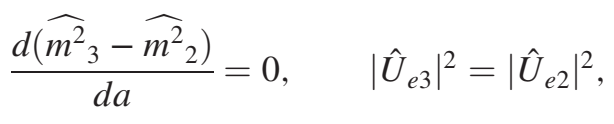

which implies $\sin ^{2} \hat{\theta}_{13} \approx 0.5$. For IO

$$
\frac{d\left(\widehat{m^{2}}{ }_{3}-\widehat{m^{2}}{ }_{1}\right)}{d a}=0, \quad\left|\hat{U}_{e 3}\right|^{2}=\left|\hat{U}_{e 1}\right|^{2}
$$

which also implies $\sin ^{2} \hat{\theta}_{13} \approx 0.5$, [30]. That is, almost maximal mixing between $\left|\hat{\nu}_{3}\right\rangle$ and $\left|\hat{\nu}_{2}\right\rangle\left(\left|\hat{\nu}_{1}\right\rangle\right)$ for NO (IO). For both NO and IO, the fractional difference between $\sin ^{2} \hat{\theta}_{13}$ and 0.5 is $\sim 2 \times 10^{-4}$ with these definitions.

However, we present here a mass ordering independent definition of the atmospheric resonance, given by

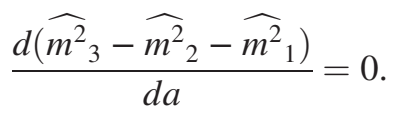

This gives an excellent approximation ${ }^{6}$ to Eq. (44) for NO as the mass of the matter eigenstate $\left|\hat{\nu}_{1}\right\rangle$ is essentially independent of the matter potential at the atmospheric resonance as $d \widehat{m}^{2}{ }_{1} / d a=\left|\hat{U}_{e 1}\right|^{2} \approx 2 \times 10^{-4}$ here. Similarly for Eq. (45) for IO.

This mass ordering independent definition also gives

$$
\left|\hat{U}_{e 3}\right|^{2}=\left|\hat{U}_{e 2}\right|^{2}+\left|\hat{U}_{e 1}\right|^{2}, \quad \sin ^{2} \hat{\theta}_{13}=0.5
$$

from Eq. (42), independent of the mass ordering. That is, exact maximal mixing between the matter eigenstate $\left|\hat{\nu}_{3}\right\rangle$ and the state $\cos \hat{\theta}_{12}\left|\hat{\nu}_{1}\right\rangle+\sin \hat{\theta}_{12}\left|\hat{\nu}_{2}\right\rangle$. Therefore, the mass ordering independent definition, Eq. (46), is what will be used for the atmospheric resonance in the rest of this paper.

Independent of this discussion, it was argued in [31] that combination $\left(\widehat{m^{2}}{ }_{3}-\widehat{m^{2}}{ }_{2}-\widehat{m^{2}}{ }_{1}\right)$ is the effective $\Delta m_{e e}^{2}(a)$ in matter with the addition of a constant that depends on one's conventions: $\Delta m_{e e}^{2}(a)$ gives the effective frequency for a $\nu_{e}$ disappearance oscillations in matter at the atmospheric minima and coincides with $\Delta m_{e e}^{2}$ in vacuum, i.e., $\Delta m_{e e}^{2}(a=0)=\Delta m_{e e}^{2}$. In the conventions of this paper,

\footnotetext{
${ }^{6}$ The fractional difference between the solution to Eqs. (44) and (46) or Eqs. (45) and (46) is $7 \times 10^{-5}$. Using Eqs. (44) and (45) as the definition of the atmospheric resonance does not change our conclusions.
} 


$$
\begin{aligned}
\Delta m_{e e}^{2}(a) & \equiv{\widehat{m^{2}}}_{3}-\widehat{m^{2}}{ }_{2}-\widehat{m^{2}}{ }_{1}+\Delta m_{21}^{2} c_{12}^{2}, \\
& \approx \Delta m_{e e}^{2} \sqrt{\left(1-2 \cos 2 \theta_{13}\left(a / \Delta m_{e e}^{2}\right)+\left(a / \Delta m_{e e}^{2}\right)^{2}\right.} .
\end{aligned}
$$

Note, this approximation is just the leading terms in $\Delta m_{e e}^{2} \mathcal{R}_{\text {atm }}$.

With the above definitions, the exact position of the solar and atmospheric resonances, $a_{\mathrm{xsol}}$ and $a_{\mathrm{xatm}}$ respectively, can be calculated perturbatively, see Appendix E, and numerical from Eq. (13) and we find that

$$
\begin{aligned}
\frac{a_{\mathrm{xsol}}}{\Delta m_{21}^{2}} & =\frac{\cos 2 \theta_{12}}{c_{13}^{2}}+\cos ^{2} 2 \theta_{12}\left(s_{13}^{2} \epsilon\right)+\cdots \\
& =\frac{\cos 2 \theta_{12}}{c_{13}^{2}}+\left\{\begin{array}{ll}
9.7 \times 10^{-5} & \text { NO } \\
-8.2 \times 10^{-5} & \text { IO }
\end{array},\right.
\end{aligned}
$$

and

$$
\begin{aligned}
\frac{a_{\text {xatm }}}{\Delta m_{e e}^{2}} & =\cos 2 \theta_{13}-s_{12}^{2} c_{12}^{2}\left(1-2 s_{13}\right)\left(\epsilon^{2}\right)+\cdots \\
& =\cos 2 \theta_{13}- \begin{cases}1.52 \times 10^{-4} & \text { NO } \\
1.49 \times 10^{-4} & \text { IO }\end{cases}
\end{aligned}
$$

Thus the accuracy of the leading terms is at the $10^{-4}$ level, provided one includes the $c_{13}^{2}$ for the solar resonance and $\Delta m_{e e}^{2}$ instead of $\Delta m_{31}^{2}$ or $\Delta m_{32}^{2}$ for the atmospheric resonance [11,32]. The magnitude of $\Delta m_{31}^{2}$ and $\Delta m_{32}^{2}$ differs from $\Delta m_{e e}^{2}$ at the $1 \%-2 \%$ level.

Comparing the Eqs. (39) and (49) and Eqs. (40) and (50), it is clear that the minima of $\mathcal{R}_{\odot}$ and $\mathcal{R}_{\text {atm }}$ do not exactly coincide with the exact solar and exact atmospheric resonances but the difference is at the $0.06 \%$ for solar and $0.005 \%$ for atmospheric which are negligible for phenomenological purposes. The order of magnitude difference between these two is probably due to the fact that the variable $\left(\widehat{m^{2}}{ }_{3}-\widehat{m^{2}}{ }_{2}-\widehat{m^{2}}{ }_{1}\right)$ is more the symmetric about the atmosphere resonance than $\left(\widehat{m^{2}}{ }_{2}-\widehat{m^{2}}{ }_{1}\right)$ is about the solar resonance, see Fig. 2.

It is also worth noting that by using the $\left.\Delta m_{e e}^{2}\right|_{\mathrm{IO}}=$ $-\left.\Delta m_{e e}^{2}\right|_{\mathrm{NO}}$ rule for switching between the two mass orderings (NO $\leftrightarrow \mathrm{IO})$, the fractional difference between $\left|a_{\text {xatm }}\right|$ for the two orderings is $\sim 3 \times 10^{-6}$ and in fact, the fractional difference between

$$
\begin{gathered}
-\left.\left({\widehat{m^{2}}}_{3}-{\widehat{m^{2}}}_{2}-{\widehat{m^{2}}}_{1}+\Delta m_{21}^{2} c_{12}^{2}\right)\right|_{\mathrm{IO}}(-a) \text { and } \\
\left.\left(\widehat{m^{2}}{ }_{3}-{\widehat{m^{2}}}_{2}-{\widehat{m^{2}}}_{1}+\Delta m_{21}^{2} c_{12}^{2}\right)\right|_{\mathrm{NO}}(a)
\end{gathered}
$$

is smaller than $10^{-5}$ for all $a$. As compared to $\sim 1 \%$, if one uses any of the following rules to flip the mass ordering:

$$
\begin{aligned}
& \left.\Delta m_{31}^{2}\right|_{\mathrm{IO}}=-\left.\Delta m_{31}^{2}\right|_{\mathrm{NO}} \quad \text { or }\left.\quad \Delta m_{32}^{2}\right|_{\mathrm{IO}}=-\left.\Delta m_{32}^{2}\right|_{\mathrm{NO}} \quad \text { or } \\
& \left.\Delta m_{32}^{2}\right|_{\mathrm{IO}}=-\left.\Delta m_{31}^{2}\right|_{\mathrm{NO}} .
\end{aligned}
$$

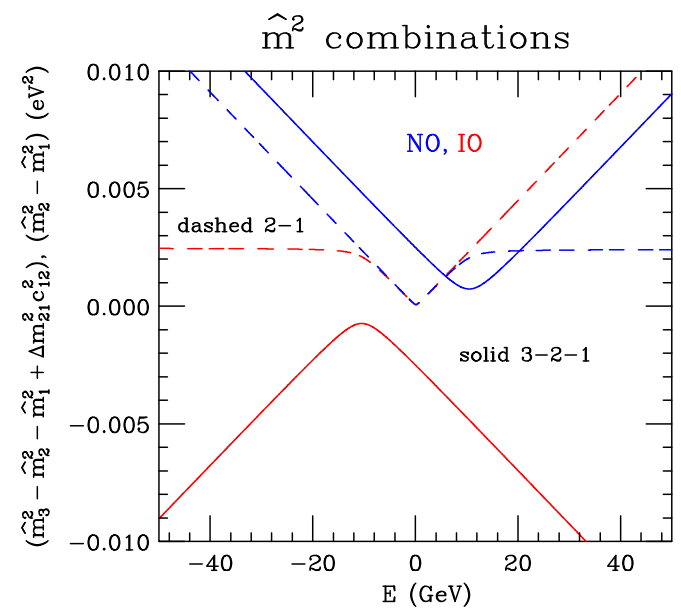

FIG. 2. The solid lines are $\left(\widehat{m^{2}}{ }_{3}-\widehat{m^{2}}{ }_{2}-\widehat{m^{2}}{ }_{1}+\Delta m_{21}^{2} c_{12}^{2}\right)$ for both NO (blue) and IO (red) as a function of the neutrino energy. Note, the approximate symmetry about the minima, $|E| \sim 10.5 \mathrm{GeV}$, for these two functions. At $E=0$, i.e., vacuum, this combination is exactly $\pm \Delta m_{e e}^{2}$, plus NO, minus for IO. The dashed lines are $\left(\widehat{m^{2}}{ }_{2}-\widehat{m^{2}}{ }_{1}\right)$ for NO (blue) and IO (red). These two functions have a significant asymmetry about the minima, $\mathrm{E} \sim 0.12 \mathrm{GeV}$, especially for $|E|>10 \mathrm{GeV}$. Here, $Y_{e} \rho=1.5$ g. $\mathrm{cm}^{-3}$. Positive (negative) energy is for neutrinos (antineutrinos) and zero is vacuum for both neutrinos and antineutrinos and all energies.

\section{SUMMARY AND CONCLUSIONS}

In this paper we have derived three new results for three flavor neutrino oscillations in matter. First, we have shown that any quantity that can be represented by a symmetric polynomial of the eigenvalues of the Hamiltonian, $\widehat{m^{2}}$, can be written as a polynomial in the matter potential, $a$. Examples of such quantities are

$$
\begin{aligned}
& \left(\prod_{j>k} \Delta{\widehat{m^{2}}}_{j k}\right)^{2}, \quad\left(\frac{J}{\hat{J}}\right)^{2} \\
& \text { and }\left(\prod_{j>k} \Delta{\widehat{m^{2}}}_{j k}\right)^{2} \times\left(\prod_{i}\left|\hat{U}_{\alpha i}\right|^{2}\right)
\end{aligned}
$$

The first two are fourth order polynomials in the matter potential whereas the last one is a constant for $\alpha=e$ and a quadratic polynomial in the matter potential for $\alpha=\mu, \tau$. Clearly there are many other such quantities that are symmetric in the eigenvalues.

Second, for the Jarlskog invariant in matter we give the exact factorization of the fourth order polynomial, such that

$$
\hat{J}=\frac{J}{\mathcal{R}_{\odot}^{\mathrm{ex}} \mathcal{R}_{\mathrm{atm}}^{\mathrm{ex}}},
$$

where 


$$
\begin{gathered}
\mathcal{R}_{\odot}^{\mathrm{ex}}=\sqrt{1-2 S_{\mathrm{sol}}^{\mathrm{ex}}\left(\frac{a}{\Delta m_{21}^{2}}\right)+T_{\mathrm{sol}}^{\mathrm{ex}}\left(\frac{a}{\Delta m_{21}^{2}}\right)^{2}}, \\
\mathcal{R}_{\mathrm{atm}}^{\mathrm{ex}}=\sqrt{1-2 S_{\mathrm{atm}}^{\mathrm{ex}}\left(\frac{a}{\Delta m_{e e}^{2}}\right)+T_{\mathrm{atm}}^{\mathrm{ex}}\left(\frac{a}{\Delta m_{e e}^{2}}\right)^{2}} .
\end{gathered}
$$

The exact coefficients $S_{\mathrm{sol}}^{\mathrm{ex}}$ to $T_{\mathrm{atm}}^{\mathrm{ex}}$ are given in Eqs. (24)(27). Since the exact coefficients involve the solution to a cubic polynomial which are notoriously challenging to penetrate analytically, a Taylor series expansion of these coefficients to $\mathcal{O}\left(s_{13}^{4} \epsilon\right), \mathcal{O}\left(s_{13}^{2} \epsilon^{2}\right), \mathcal{O}\left(\epsilon^{3}\right)$, is also given in Eqs. (32)-(35). The fractional accuracy of these coefficients is better than $10^{6}$, as shown in Table I.

Third, we define the solar resonance to be when

$$
\frac{d\left(\widehat{m^{2}}{ }_{2}-\widehat{m^{2}}{ }_{1}\right)}{d a}=0 \Leftrightarrow \sin ^{2} \hat{\theta}_{12}=0.5,
$$

and calculate that this occurs when

$$
\frac{a}{\Delta m_{21}^{2}}=\frac{\cos 2 \theta_{12}}{c_{13}^{2}}+\cos ^{2} 2 \theta_{12}\left(s_{13}^{2} \epsilon\right)+\cdots,
$$

where the "..." are higher order terms in $s_{13}^{2}$ and $\epsilon \equiv \Delta m_{21}^{2} / \Delta m_{e e}^{2}$. Independent of the mass ordering, we define the atmospheric resonance to be when

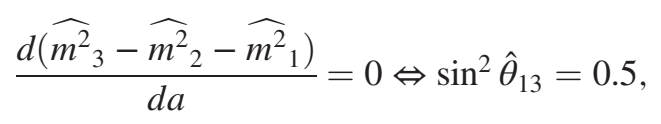

and calculate that this occurs when

$$
\frac{a}{\Delta m_{e e}^{2}}=\cos 2 \theta_{13}-s_{12}^{2} c_{12}^{2}\left(1-2 s_{13}\right)\left(\epsilon^{2}\right)+\cdots .
$$

For three flavors of neutrinos, these are the most accurate values for the locations of the solar and atmospheric resonances available in the literature. The value of the matter potential typically quoted for the atmospheric resonance, $a / \Delta m_{32}^{2}=\cos 2 \theta_{13}$, differs from above value by $2 \%$, a phenomenological significant difference.

We have also made a comparison between the location of the minima in the matter potential for the two factorizing factors, $\mathcal{R}_{\odot}^{\mathrm{ex}}$ and $\mathcal{R}_{\mathrm{atm}}^{\mathrm{ex}}$, and the location in matter potential of the solar and atmospheric resonances. The difference in the value of the matter potential between the location of the minima and the resonance values are $0.06 \%$ for solar and $0.005 \%$ for atmospheric, therefore they do not coincide. However, these difference are negligible for phenomenological purposes.

To conclude, the understanding of the physics of neutrino propagation in matter is of essential importance to the current NOvA and T2K, and upcoming DUNE and $\mathrm{T} 2 \mathrm{HK}(\mathrm{K})$ long baseline neutrino oscillation experiments. The Wolfenstein matter effect significantly complicates three flavor neutrino oscillations in matter compared to vacuum. The exact analytic expressions, even for constant matter density, are analytically impenetrable due to the presence of the $\cos \left\{\frac{1}{3} \cos ^{-1}[\cdots]\right\}$ terms. In this paper we show that variables that depend on the symmetric polynomials of the mass squared of the neutrino eigenstates in matter are just simple polynomials of the matter potential, a significant simplification. We give a number of examples and in particular the Jarlskog invariant in matter which controls the size of intrinsic $C P$ violation in the above neutrino appearance experiments. We give for the first time the exact factorization of the Jarlskog invariant in matter into two quadratic resonance factors. The location of the minima of these resonance factors is compared to the location of the solar and atmospheric resonances to the appropriate order in the small quantities $s_{13}^{2}$ and $\Delta m_{21}^{2} / \Delta m_{e e}^{2}$. This required, for the first time, the calculation of the location of the solar and atmospheric resonances beyond leading order. All of this further enhances our understanding of neutrino propagation in matter relevant for the long baseline neutrino oscillation experiments being currently performed and for the next generation of experiments.

\section{ACKNOWLEDGMENTS}

I thank Peter Denton for many enlightening discussions during the course of this work. Fermilab is operated by the Fermi Research Alliance under Contract No. DE-AC0207CH11359 with the U.S. Department of Energy. This project has received funding/support from the European Union's Horizon 2020 research and innovation program under the Marie Sklodowska-Curie Grant Agreement No. 860881-HIDDeN.

\section{APPENDIX A: THE VARIABLES $\xi$ AND $\chi$}

The quantities $(\xi+\chi)_{\alpha}$ and $(\xi \chi)_{\alpha}$ are the trace and determinant of the submatrix of (2E)H with the $\alpha$ row and $\alpha$ column removed. In the flavor basis, since $H_{e e}$ is the only element that depends on the matter potential these traces and determinants are at most linear polynomials in the matter potential, $a$. In fact, $(\xi+\chi)_{e}$ and $(\xi \chi)_{e}$ are independent of the matter potential. Explicitly,

$$
\begin{aligned}
\xi_{e}+\chi_{e}= & \left(\mathcal{H}_{\mu \mu}+\mathcal{H}_{\tau \tau}\right)=\Delta m_{31}^{2} c_{13}^{2}+\Delta m_{21}^{2}\left(c_{12}^{2}+s_{13}^{2} s_{12}^{2}\right) \\
= & d B / d a, \\
\xi_{e} \chi_{e}= & \left(\mathcal{H}_{\mu \mu} \mathcal{H}_{\tau \tau}-\mathcal{H}_{\mu \tau}^{2}\right)=\Delta m_{31}^{2} \Delta m_{21}^{2} c_{13}^{2} c_{12}^{2}=d C / d a, \\
\xi_{\mu}+\chi_{\mu}= & {\left[c_{23}^{2}\left(\mathcal{H}_{e e}+\mathcal{H}_{\tau \tau}\right)+s_{23}^{2}\left(\mathcal{H}_{e e}+\mathcal{H}_{\mu \mu}\right)\right.} \\
& \left.-2 s_{23} c_{23} \cos \delta\left(\mathcal{H}_{\mu \tau}\right)\right], \\
\xi_{\mu} \chi_{\mu}= & {\left[c_{23}^{2}\left(\mathcal{H}_{e e} \mathcal{H}_{\tau \tau}-\mathcal{H}_{e \tau}^{2}\right)+s_{23}^{2}\left(\mathcal{H}_{e e} \mathcal{H}_{\mu \mu}-\mathcal{H}_{e \mu}^{2}\right)\right.} \\
& \left.-2 s_{23} c_{23} \cos \delta\left(\mathcal{H}_{e e} \mathcal{H}_{\mu \tau}-\mathcal{H}_{e \mu} \mathcal{H}_{e \tau}\right)\right] .
\end{aligned}
$$


The $\xi_{\tau}$ and $\chi_{\tau}$ eigenvalues are the same as $\xi_{\mu}$ and $\chi_{\mu}$ under the interchange $s_{23}^{2} \leftrightarrow c_{23}^{2}$ and $s_{23} c_{23} \rightarrow-s_{23} c_{23}$. Where

$$
\mathcal{H}=\left(\begin{array}{ccc}
a+\Delta m_{e e}^{2} s_{13}^{2}+\Delta m_{21}^{2} s_{12}^{2} & c_{13} s_{12} c_{12} \Delta m_{21}^{2} & s_{13} c_{13} \Delta m_{e e}^{2} \\
\cdot & \Delta m_{21}^{2} c_{12}^{2} & -s_{13} s_{12} c_{12} \Delta m_{21}^{2} \\
\cdot & \cdot & \Delta m_{e e}^{2} c_{13}^{2}+\Delta m_{21}^{2} s_{12}^{2}
\end{array}\right),
$$

where $\mathcal{H}_{\alpha \beta}=\mathcal{H}_{\beta \alpha}$ and $\Delta m_{e e}^{2} \equiv c_{12}^{2} \Delta m_{31}^{2}+s_{12}^{2} \Delta m_{31}^{2}$ [29]. The relationship between $\mathcal{H}$ and $\mathrm{H}$ of Eq. (7) is $\mathcal{H}=$ $(2 E) U_{23}^{\dagger}\left(\theta_{23}, \delta\right) H U_{23}\left(\theta_{23}, \delta\right)$. See [25] for further details.

Defining $\xi_{\alpha} \leq \chi_{\alpha}$, then the Cauchy interlace theorem tells us that $\widehat{m^{2}}{ }_{1} \leq \xi_{\alpha} \leq{\widehat{m^{2}}}_{2} \leq \chi_{\alpha} \leq \widehat{m^{2}}{ }_{3}$ for NO.

\section{APPENDIX B: EVALUATION OF$$
\prod_{i}\left(\left({\widehat{\boldsymbol{m}^{2}}}_{i}\right)^{2}-(\xi+\chi)_{\alpha}{\widehat{\boldsymbol{m}^{2}}}_{i}+(\xi \chi)_{\alpha}\right)
$$

It is straightforward to show that

$$
\begin{aligned}
& \prod_{i}\left(\left(\widehat{m^{2}}{ }_{i}\right)^{2}-(\xi+\chi)_{\alpha} \widehat{m^{2}}{ }_{i}+(\xi \chi)_{\alpha}\right) \\
& =C\left[C-B(\xi+\chi)_{\alpha}+A(\xi+\chi)_{\alpha}^{2}-(\xi+\chi)_{\alpha}^{3}\right] \\
& \quad-(\xi+\chi)_{\alpha}(\xi \chi)_{\alpha}\left[(A B-3 C)-B(\xi+\chi)_{\alpha}+A(\xi \chi)_{\alpha}\right] \\
& \quad+(\xi \chi)_{\alpha}\left[\left(B^{2}-2 A C\right)+\left(A^{2}-2 B\right)(\xi \chi)_{\alpha}+(\xi \chi)_{\alpha}^{2}\right] .
\end{aligned}
$$

For $\alpha=e$, both $(\xi+\chi)_{e}$ and $(\xi \chi)_{e}$ are independent of $a$ and this is at most a quadratic polynomial in $a$. The quadratic and linear terms in $a$ cancel and one is left with the constant, so this expression is independent of the matter potential.

For $\alpha=\mu$ (or $\tau$ ), both $(\xi+\chi)_{\mu}$ and $(\xi \chi)_{\mu}$ are linear in $a$ and this polynomial is at most fourth order in $a$. Again the top two powers of $a$ cancel and one is left with a quadratic polynomial in $a$. Since $(\xi+\chi)_{\mu}$ and $(\xi \chi)_{\mu}$ depend on $\theta_{23}$ and $\delta$ it is convenient to reorganize the calculation by multiplying $\left(\widehat{m^{2}}{ }_{i}\right)^{2}$ by $\left(c_{23}^{2}+s_{23}^{2}\right)$ as follows:

$$
\begin{aligned}
& \prod_{i}\left(\left(\widehat{m^{2}}{ }_{i}\right)^{2}-(\xi+\chi)_{\mu}{\widehat{m^{2}}}_{i}+(\xi \chi)_{\mu}\right) \\
& \quad=\prod_{i}\left(c_{23}^{2} X_{i}+s_{23}^{2} Y_{i}+2 s_{23} c_{23} \cos \delta Z_{i}\right)
\end{aligned}
$$

where

$$
\begin{aligned}
& X_{i}=\left(\widehat{m^{2}}{ }_{i}\right)^{2}-\left(\mathcal{H}_{e e}+\mathcal{H}_{\tau \tau}\right)\left(\widehat{m^{2}}{ }_{i}\right)+\left(\mathcal{H}_{e e} \mathcal{H}_{\tau \tau}-\mathcal{H}_{e \tau}{ }_{e}\right)=\left(\widehat{m^{2}}{ }_{i}-\mathcal{H}_{e e}\right)\left(\widehat{m}^{2}{ }_{i}-\mathcal{H}_{\tau \tau}\right)-\mathcal{H}_{e \tau}{ }^{2}, \\
& Y_{i}=\left({\widehat{m^{2}}}_{i}\right)^{2}-\left(\mathcal{H}_{e e}+\mathcal{H}_{\mu \mu}\right)\left(\widehat{m}^{2}{ }_{i}\right)+\left(\mathcal{H}_{e e} \mathcal{H}_{\mu \mu}-\mathcal{H}_{e \mu}^{2}\right)=\left({\widehat{m^{2}}}_{i}-\mathcal{H}_{e e}\right)\left(\widehat{m}^{2}{ }_{i}-\mathcal{H}_{\mu \mu}\right)-\mathcal{H}_{e \mu}^{2} \text {, } \\
& Z_{i}=\mathcal{H}_{\mu \tau}\left({\widehat{m^{2}}}_{i}\right)-\left(\mathcal{H}_{e e} \mathcal{H}_{\mu \tau}-\mathcal{H}_{e \mu} \mathcal{H}_{e \tau}\right)=\mathcal{H}_{\mu \tau}\left({\widehat{m^{2}}}_{i}-\mathcal{H}_{e e}\right)-\mathcal{H}_{e \mu} \mathcal{H}_{e \tau} \text {, }
\end{aligned}
$$

which satisfy the following condition that $X_{i} Y_{i}=Z_{i}^{2}$. Then the left-hand side of Eq. (B1) is a homogeneous third order polynomial in $c_{23}^{2}, s_{23}^{2}$ and $\left(2 s_{23} c_{23} \cos \delta\right)$ :

$$
\begin{aligned}
\prod_{i}\left(c_{23}^{2} X_{i}+s_{23}^{2} Y_{i}+2 s_{23} c_{23} \cos \delta Z_{i}\right)= & c_{23}^{6}\left[X_{1} X_{2} X_{3}\right]+c_{23}^{4} s_{23}^{2}\left[X_{1} X_{2} Y_{3}\right]+c_{23}^{2} s_{23}^{4}\left[X_{1} Y_{2} Y_{3}\right]+s_{23}^{6}\left[Y_{1} Y_{2} Y_{3}\right] \\
& +\left(2 s_{23} c_{23} \cos \delta\right)\left(c_{23}^{4}\left[Z_{1} X_{2} X_{3}\right]+c_{23}^{2} s_{23}^{2}\left[X_{1} Y_{2} Z_{3}\right]+s_{23}^{4}\left[Z_{1} Y_{2} Y_{3}\right]\right) \\
& +\left(2 s_{23} c_{23} \cos \delta\right)^{2}\left(c_{23}^{2}\left[Z_{1} Z_{2} X_{3}\right]+s_{23}^{2}\left[Z_{1} Z_{2} Y_{3}\right]\right)+\left(2 s_{23} c_{23} \cos \delta\right)^{3}\left[Z_{1} Z_{2} Z_{3}\right]
\end{aligned}
$$

where each $[\cdots]$ is symmeterized over the labels $(1,2,3)$ which guarantees that each term is a polynomial in $a$. This is easy to calculate using an algebraic program like FORM. Some, but not all of the terms are very simple; we give here examples of the simplest terms.

The coefficient of $s_{23}^{6}$ is given by

$$
Y_{1} Y_{2} Y_{3}=s_{13}^{4} c_{13}^{2} s_{12}^{2} c_{12}^{2}\left(\Delta m_{21}^{2}\right)^{2}\left(\Delta m_{31}^{2} \Delta m_{32}^{2}+a \Delta m_{e e}^{2}\right)^{2},
$$

the coefficient of $c_{23}^{6}$ is

$$
X_{1} X_{2} X_{3}=s_{13}^{2} c_{13}^{2} s_{12}^{4} c_{12}^{4}\left(\Delta m_{21}^{2}\right)^{4} a^{2},
$$

and the coefficient of $\left(2 s_{23} c_{23} \cos \delta\right)^{3}$

$$
Z_{1} Z_{2} Z_{3}=s_{13}^{3} c_{13}^{2} s_{12}^{3} c_{12}^{3}\left(\Delta m_{21}^{2}\right)^{3}\left(\Delta m_{31}^{2} \Delta m_{32}^{2}+a \Delta m_{e e}^{2}\right) a .
$$

Note $Z_{1} Z_{2} Z_{3}=\sqrt{X_{1} X_{2} X_{3} Y_{1} Y_{2} Y_{3}}$.

For $\alpha=\tau$, just interchange $s_{23}^{2} \leftrightarrow c_{23}^{2}$ and flip the sign of $s_{23} c_{23}$. 


\section{APPENDIX C: DERIVATION OF THE $f_{n}$ 's}

Writing $A, B$, and $C$, defined in Eq. (12), as constant plus linear in $a$ terms, reads as follows:

$$
\begin{aligned}
& A=A_{0}+a, \quad A_{0}=\Delta m_{21}^{2}+\Delta m_{31}^{2}, \\
& B=B_{0}+a B_{1}, \quad B_{0}=\Delta m_{31}^{2} \Delta m_{21}^{2}, \quad B_{1}=\Delta m_{31}^{2} c_{13}^{2}+\Delta m_{21}^{2}\left(c_{12}^{2}+s_{13}^{2} s_{12}^{2}\right), \\
& C=a C_{1}, \quad C_{1}=\Delta m_{31}^{2} \Delta m_{21}^{2} c_{13}^{2} c_{12}^{2} .
\end{aligned}
$$

Then from Eq. (15), the $f_{n}$ 's of Eq. (21) are given by

$$
\begin{aligned}
& f_{1}=2 \Delta m_{21}^{2}\left[\left(A_{0}^{2}-4 B_{0}\right)\left(B_{0} B_{1}-2 A_{0} C_{1}\right)+B_{0}^{2}\left(A_{0}-2 B_{1}\right)+A_{0} B_{0} C_{1}\right] / f_{0}, \\
& f_{2}=\left(\Delta m_{21}^{2}\right)^{2}\left[\left(A_{0}^{2}-4 B_{0}\right)\left(B_{1}^{2}-4 C_{1}\right)+B_{0}^{2}+4\left(A_{0}-2 B_{1}\right)\left(B_{0} B_{1}-2 A_{0} C_{1}\right)+2\left(B_{1} A_{0}+B_{0}\right) C_{1}-27 C_{1}^{2}\right] / f_{0}, \\
& f_{3}=2\left(\Delta m_{21}^{2}\right)^{3}\left[\left(B_{0} B_{1}-2 A_{0} C_{1}\right)+\left(B_{1}^{2}-4 C_{1}\right)\left(A_{0}-2 B_{1}\right)+B_{1} C_{1}\right] / f_{0}, \\
& f_{4}=\left(\Delta m_{21}^{2}\right)^{4}\left(B_{1}^{2}-4 C_{1}\right) / f_{0},
\end{aligned}
$$

where $f_{0}=B_{0}^{2}\left(A_{0}^{2}-4 B_{0}\right)=\left(\Delta m_{21}^{2} \Delta m_{31}^{2} \Delta m_{32}^{2}\right)^{2}$.

Note, $f_{1}, f_{2}, f_{3}$, and $f_{4}$ are of order $1,1, \epsilon$, and $\epsilon^{2}$, respectively, where $\epsilon \equiv \Delta m_{21}^{2} / \Delta m_{e e}^{2}$.

\section{APPENDIX D: PROOF OF ${\boldsymbol{d m ^ { 2 }}}_{i} / \boldsymbol{d a}=\left|\hat{U}_{e i}\right|^{2}$}

Differentiate $A, B$, and $C$ of Eq. (12) with respect to $a$, to obtain the following:

$$
\begin{aligned}
& 1=\frac{\widehat{d m^{2}}}{d a}+\frac{\widehat{d m^{2}} 2}{d a}+\frac{d m_{2}^{2}}{d a}, \\
& (\xi+\chi)_{e}=\left(\widehat{m^{2}}{ }_{2}+{\widehat{m^{2}}}_{3}\right) \frac{d \widehat{m}^{2}}{d a}+\left(\widehat{m^{2}}{ }_{3}+{\widehat{m^{2}}}_{1}\right) \frac{d \widehat{m}^{2}}{d a} \\
& +\left(\widehat{m^{2}}{ }_{1}+{\widehat{m^{2}}}_{2}\right) \frac{d \bar{m}^{2}}{d a}, \\
& (\xi \chi)_{e}=\left(\widehat{m^{2}}{ }_{2}{\widehat{m^{2}}}_{3}\right) \frac{d \bar{m}^{2}}{d a}+\left(\widehat{m^{2}}{ }_{3}{\widehat{m^{2}}}_{1}\right) \frac{d \bar{m}^{2}}{d a} \\
& +\left(\widehat{m^{2}} \widehat{m}_{1}^{2}\right) \frac{d m^{2}}{d a} \text {. }
\end{aligned}
$$

Multiplying Eq. (D1) by $\left(\widehat{m^{2}}{ }^{2}\right)^{2}$, Eq. (D2) by $\left(-\widehat{m^{2}}{ }_{i}\right)$, and add these to Eq. (D3), one obtains

$\frac{d \widehat{m}^{2}}{d a}=\frac{\left({\widehat{m^{2}}}_{i}\right)^{2}-(\xi+\chi)_{e}\left({\widehat{m^{2}}}_{i}\right)+(\xi \chi)_{e}}{\Delta{\widehat{m^{2}}}{ }_{i j} \Delta{\widehat{m^{2}}}{ }_{i k}}=\left|\widehat{U}_{e i}\right|^{2}$,

with the indices $i, j$, and $k$ all different. See Eq. (16).

\section{APPENDIX E: PERTURBATIVE EXPANSIONS ABOUT THE RESONANCES}

A perturbative expansion is used to obtain the values of the matter potential for the atmospheric and solar resonances.

\section{Atmospheric resonance}

In this appendix we perform an perturbative expansion in the matter potential about the approximate value of the matter potential that minimizes $\left(\widehat{m^{2}}{ }_{3}-\widehat{m^{2}}{ }_{2}-\widehat{m^{2}}{ }_{1}\right)$, that is

$$
a=\Delta m_{e e}^{2} \cos 2 \theta_{13}+\delta a
$$

Starting with the Hamiltonian from Eq. (7), a (2-3) rotation using $U_{23}\left(\theta_{23}, \delta\right)$ is performed to remove the $\theta_{23}$ and $\delta$ dependence. Then a $\pi / 4$ rotation is performed in the (1-3) sector using the value of $a$ given in Eq. (E1). The resulting Hamiltonian is given by

$U_{13}^{\dagger}(\pi / 4) U_{23}^{\dagger}\left(\theta_{23}, \delta\right)(2 E) H U_{23}\left(\theta_{23}, \delta\right) U_{13}(\pi / 4)=H_{a}+h$

with

$$
\begin{aligned}
H_{a}= & \operatorname{diag}\left(\Delta m_{e e}^{2}\left(c_{13}^{2}-s_{13} c_{13}\right)+\Delta m_{12}^{2} s_{12}^{2},\right. \\
& \left.\Delta m_{21}^{2} c_{12}^{2}, \quad \Delta m_{e e}^{2}\left(c_{13}^{2}+s_{13} c_{13}\right)+\Delta m_{12}^{2} s_{12}^{2}\right),
\end{aligned}
$$

$$
h=\frac{1}{2}\left(\begin{array}{ccc}
\delta a & \delta m_{+}^{2} & \delta a \\
\cdot & 0 & \delta m_{-}^{2} \\
\cdot & \cdot & \delta a
\end{array}\right)
$$

where $\delta m_{ \pm}^{2}=\sqrt{2} \Delta m_{21}^{2} s_{12} c_{12}\left(c_{13} \pm s_{13}\right)$. Note, because the solar crossing has not been resolved the mass eigenstates $\nu_{1}$ and $\nu_{2}$ are interchanged. Since $h$ is symmetric, the ...'s are given by the appropriate term above the diagonal.

Now perturbation theory can be used to calculate $\left(\widehat{m^{2}}{ }_{3}-\widehat{m^{2}}{ }_{2}-\widehat{m^{2}}{ }_{1}\right)$, which to third order in $h$, is given by 


$$
\begin{aligned}
\widehat{m^{2}}{ }_{3}-\widehat{m^{2}}{ }_{2}-\hat{m}_{1}^{2} \approx & d_{31}+2\left(h_{13}^{2} / d_{31}+h_{23}^{2} / d_{32}\right) \\
& +2 h_{11} h_{23}\left(2 h_{12} d_{32}-h_{23} d_{31}\right) /\left(d_{32}^{2} d_{31}\right),
\end{aligned}
$$

where $h_{j k}$ are the elements of matrix $h$, Eq. (E4), and $d_{j k}=H_{a}(j, j)-H_{a}(k, k), \quad$ e.g., $\quad d_{31}=\Delta m_{e e}^{2}\left(2 s_{13} c_{13}\right)$. Equation (E5) is a quadratic in the variable $\delta a$ as both $h_{11}$ and $h_{13}$ are linear in $\delta a$. To obtain an estimate of the shift one needs to go to third order, as first order vanishes, second order is quadratic, and third order gives the first odd term in $\delta a$. The value of $\delta a$ that minimizes $\left(\widehat{m^{2}}{ }_{3}-\widehat{m^{2}}{ }_{2}-\widehat{m^{2}}{ }_{1}\right)$ is given by

$$
\begin{aligned}
\delta a & =-h_{23}\left(2 h_{12} d_{32}-h_{23} d_{31}\right) / d_{32}^{2} \\
& \approx-s_{12}^{2} c_{12}^{2}\left(1-2 s_{13}\right)\left(\Delta m_{21}^{2}\right)^{2} / \Delta m_{e e}^{2} .
\end{aligned}
$$

This gives Eq. (50). The minimum of Eq. (E5) reproduces the exact minimum to better than one part in $10^{8}$.

Using the above perturbation theory, one can also show that the value of $\delta a$ that minimizes $\left(\widehat{m^{2}}{ }_{3}-\widehat{m^{2}}{ }_{2}\right)$ for NO is given by

$$
\delta a \approx-s_{12}^{2} c_{12}^{2}\left(\Delta m_{21}^{2}\right)^{2} / \Delta m_{e e}^{2} .
$$

Thus the alternative definition of the atmospheric resonance, Eq. (44), changes the corrections at $\mathcal{O}\left(s_{13} \epsilon^{2}\right)$ and similarly for IO.

\section{Solar resonance}

In this appendix we perform a perturbative expansion in the matter potential about the approximate value of the matter potential that minimizes $\left(\widehat{m^{2}}{ }_{2}-\widehat{m^{2}}{ }_{1}\right)$, that is

$$
a=a_{0}+\delta a, \quad a_{0}=\Delta m_{21}^{2} \cos 2 \theta_{12} / \cos ^{2} \theta_{13} .
$$

Starting with the Hamiltonian from Eq. (7), a (2-3) rotation using $U_{23}\left(\theta_{23}, \delta\right)$ is performed to remove the $\theta_{23}$ and $\delta$ dependence. Then a $\theta_{13}$ rotation is performed in the (1-3) sector followed by a $\pi / 4$ rotation performed in the (1-2) sector using the value of $a$ given in Eq. (E8). The resulting
Hamiltonian is given by

$$
\begin{aligned}
& U_{12}^{\dagger}(\pi / 4) U_{13}^{\dagger}\left(\theta_{13}\right) U_{23}^{\dagger}\left(\theta_{23}, \delta\right)(2 E) H U_{23}\left(\theta_{23}, \delta\right) \\
& \quad \times U_{13}\left(\theta_{13}\right) U_{12}(\pi / 4)=H_{s}+h
\end{aligned}
$$

where

$$
\begin{aligned}
& H_{s}=\operatorname{diag}\left(\Delta m_{21}^{2}\left(c_{12}^{2}-s_{12} c_{12}\right), \quad \Delta m_{21}^{2}\left(c_{12}^{2}+s_{12} c_{12}\right),\right. \\
& \left.\Delta m_{31}^{2}+a_{0}\right) \text {, } \\
& h=\frac{1}{2}\left(\begin{array}{ccc}
\delta a c_{13}^{2} & \delta a c_{13}^{2} & \sqrt{2} a s_{13} c_{13} \\
\cdot & \delta a c_{13}^{2} & \sqrt{2} a s_{13} c_{13} \\
\cdot & \cdot & 2 \delta a s_{13}^{2}
\end{array}\right) .
\end{aligned}
$$

Since $h$ is symmetric, the ...'s are given by the appropriate term above the diagonal. There are only three independent elements in $h$ : we will use $h_{11}, h_{13}, h_{33}$ as the independent ones.

Now perturbation theory can be used to calculate $\left(\widehat{m^{2}}{ }_{2}-\widehat{m^{2}}{ }_{1}\right)$, which to third order in $h$ is given by

$$
\begin{aligned}
\widehat{m^{2}}{ }_{2}-\widehat{m^{2}}{ }_{1} \approx & d_{21}+2 h_{11}^{2} / d_{21}-h_{13}^{2} d_{21} /\left(d_{32} d_{31}\right) \\
& -h_{13}^{2}\left(d_{31}+d_{32}\right)\left[2 h_{11} /\left(d_{32} d_{31} d_{21}\right)\right. \\
& \left.+\left(h_{11}-h_{33}\right) d_{21} /\left(d_{31}^{2} d_{32}^{2}\right)\right]
\end{aligned}
$$

where here $h_{j k}$ are the elements of matrix $h$, Eq. (E11), and $d_{j k}=H_{s}(j, j)-H_{s}(k, k)$, e.g., $d_{21}=\Delta m_{21}^{2}\left(2 s_{12} c_{12}\right)$.

Using the fact that $h_{13}$ is approximately constant, $h_{13} \approx a_{0} s_{13} c_{13} / \sqrt{2}$, and keeping only the terms with $d_{21}$ in the denominator, then the value of $\delta a$ that minimizes $\left(\widehat{m^{2}}{ }_{2}-\widehat{m^{2}}{ }_{1}\right)$ is approximately given by

$$
\delta a \approx h_{13}^{2}\left(d_{31}+d_{32}\right) /\left(d_{31} d_{32}\right) \approx \cos ^{2} 2 \theta_{12} s_{13}^{2}\left(\Delta m_{21}^{2}\right)^{2} / \Delta m_{e e}^{2} .
$$

This gives Eq. (49). The minimum of Eq. (E12) reproduces the exact minimum to one part in $10^{7}$.
[1] Y. Itow et al. (T2K Collaboration), in Neutrino oscillations and their origin, Proceedings, 3rd International Workshop, NOON 2001, Kashiwa, Tokyo, Japan, 2001 (2001), pp. 239-248 [arXiv:hep-ex/0106019].

[2] D. S. Ayres et al. (NOvA Collaboration), arXiv:hep-ex/ 0503053.
[3] R. Acciarri et al. (DUNE Collaboration), arXiv:1601.05471.

[4] K. Abe et al. (Hyper-Kamiokande Working Group), arXiv: 1412.4673.

[5] K. Abe et al. (Hyper-Kamiokande Collaboration), Prog. Theor. Exp. Phys. (2018), 063C01.

[6] L. Wolfenstein, Phys. Rev. D 17, 2369 (1978). 
[7] S. M. Bilenky and S. T. Petcov, Rev. Mod. Phys. 59, 671 (1987); 60, 575(E) (1988).

[8] C. Jarlskog, Phys. Rev. Lett. 55, 1039 (1985).

[9] Z. Maki, M. Nakagawa, and S. Sakata, Prog. Theor. Phys. 28, 870 (1962).

[10] B. Pontecorvo, Zh. Eksp. Teor. Fiz. 53, 1717 (1967) [Sov. Phys. JETP 26, 984 (1968)].

[11] H. W. Zaglauer and K. H. Schwarzer, Z. Phys. C 40, 273 (1988).

[12] P. I. Krastev and S. T. Petcov, Phys. Lett. B 205, 84 (1988).

[13] S. J. Parke and T. J. Weiler, Phys. Lett. B 501, 106 (2001).

[14] P. B. Denton and S. J. Parke, Phys. Rev. D 100, 053004 (2019).

[15] X. Wang and S. Zhou, J. High Energy Phys. 05 (2019) 035.

[16] X. Wang and S. Zhou, Nucl. Phys. B950, 114867 (2020).

[17] P. B. Denton and R. Pestes, arXiv:2006.09384.

[18] K. J. Kelly and S. J. Parke, Phys. Rev. D 98, 015025 (2018).

[19] S. F. King, S. Molina Sedgwick, S. J. Parke, and N. W. Prouse, Phys. Rev. D 101, 076019 (2020).

[20] G. Cardano, Ars Magna (1545).
[21] V. D. Barger, K. Whisnant, S. Pakvasa, and R. J. N. Phillips, Phys. Rev. D 22, 2718 (1980).

[22] Wikipedia, Elementary symmetric polynomials (2020).

[23] P. F. Harrison and W. G. Scott, Phys. Lett. B 476, 349 (2000).

[24] H. Yokomakura, K. Kimura, and A. Takamura, Phys. Lett. B 496, 175 (2000).

[25] P. B. Denton, S. J. Parke, and X. Zhang, Phys. Rev. D 101, 093001 (2020).

[26] P. B. Denton, S. J. Parke, T. Tao, and X. Zhang, arXiv: 1908.03795.

[27] V. A. Naumov, Int. J. Mod. Phys. D 01, 379 (1992).

[28] S. Toshev, Mod. Phys. Lett. A 06, 455 (1991).

[29] H. Nunokawa, S. J. Parke, and R. Zukanovich Funchal, Phys. Rev. D 72, 013009 (2005).

[30] Z.-Z. Xing, S. Zhou, and Y.-L. Zhou, J. High Energy Phys. 05 (2018) 015.

[31] P. B. Denton and S. J. Parke, Phys. Rev. D 98, 093001 (2018).

[32] P. B. Denton, H. Minakata, and S. J. Parke, J. High Energy Phys. 06 (2016) 051. 